\title{
Topics in Electroweak Physics
}

\author{
A. $\operatorname{Sirlin}^{\mathrm{a} *}$

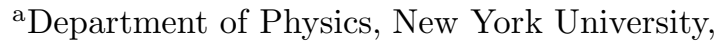 \\ 4 Washington Place, NY 10003, United States
}

We briefly discuss five topics in Precision Electroweak Physics: i) the recently proposed Effective Scheme of Renormalization, ii) evidence for electroweak bosonic corrections derived from the radiative correction $\Delta r_{\text {eff }}$, iii) an approach to estimate the scale of new physics in a hypothetical Higgs-less scenario, iv) simple and accurate formulae for $s_{e f f}^{2}, M_{W}, \Gamma_{l}$, and their physical applications, v) a recent proposal concerning the field renormalization constant for unstable particles.

\section{Effective Scheme of Renormalization}

Precise calculations in the Standard Model (SM) are based on a number of renormalization frameworks. Two of the most frequently employed are: 1) the On-Shell Scheme (OS) [13] 2) the $\overline{M S}$ approach [4]. The OS scheme is "very physical" in the sense that the renormalized parameters are identified with physical, scale-independent observables, such as $\alpha, G_{F}$, $M_{Z}, M_{W}, \ldots$ The $\overline{M S}$ approach is frequently applied in a hybrid version, with couplings defined by $\overline{M S}$ subtractions, but retaining physical masses. It employs scale-dependent parameters such as $\hat{s}^{2} \equiv \sin ^{2} \hat{\theta}(\mu), \hat{e}^{2}(\mu)$ (usually evaluated at $\mu=M_{z}$ ) and exhibits very good convergence properties [5]. It plays an important rôle in the analysis of Grand Unified Theories. However, it leads to a residual scale dependence in finite orders of perturbation theory (PT). Very recently, a novel approach, called the Effective Scheme of Renormalization (EFF), was proposed $[6,7]$. It shares the good convergence properties of the $\overline{M S}$ scheme, but it eliminates the residual scale dependence in finite orders of PT. A distinguishing feature is that the basic electroweak mixing parameter (EWMP) is directly identified with $s_{\text {eff }}^{2} \equiv \sin ^{2} \theta_{\text {eff }}^{\text {lept }}$, employed by the Electroweak Working Group (EWWG) to describe the on-resonance asymmetries measured at LEP and

${ }^{*}$ Talk presented at the International Symposium Radcor 2002, September 8-13, Kloster Banz, Germany. This work was supported in part by NSF Grant No. PHY-0070787.
SLC. It may be evaluated by means of the basic relation $[6-8]$

$$
s_{e f f}^{2} c_{e f f}^{2}=\frac{\pi \alpha}{\sqrt{2} G_{F} M_{Z}^{2}\left(1-\Delta r_{e f f}\right)},
$$

where $\Delta r_{\text {eff }}$ is the relevant radiative correction. In order to calculate $\Delta r_{\text {eff }}$ the following strategy was followed:

i) Since current calculations of $s_{\text {eff }}^{2}$ incorporate two-loop effects enhanced by powers $\left(M_{t}^{2} / M_{Z}^{2}\right)^{n}$ (with $n=1,2$ ), we first express $\Delta r_{\text {eff }}$ in terms of corrections $\Delta \hat{r}_{W}, \Delta \hat{\rho}, \Delta \hat{k}$ and $\hat{f}$, for which the irreducible contributions of this order have been evaluated $[9,10]$. ii) To ensure the absence of a residual scale dependence, we use scale-independent couplings, such as $e^{2}, s_{e f f}^{2}, G_{F}, M_{Z}^{2}$, retain only two-loop effects enhanced by factors $\left(M_{t}^{2} / M_{Z}^{2}\right)^{n}$ $(n=1,2)$, and employ a single definition of the EWMP, identified with $s_{\text {eff }}^{2}$. In particular, $\hat{s}^{2}$ can be expressed in terms of $s_{\text {eff }}^{2}$ by means of the relation

$s_{e f f}^{2}=\left[1+\frac{\hat{e}^{2}}{\hat{s}^{2}} \Delta \hat{k}\left(M_{Z}^{2}, \mu\right)\right] \hat{s}^{2}(\mu)$,

where $\Delta \hat{k}\left(q^{2}, \mu\right)$ is an electroweak form factor [11]. The analysis leads to the expression [6]:

$$
\begin{aligned}
& \Delta r_{e f f}=\Delta \hat{r}_{W}-\frac{e^{2}}{s_{e f f}^{2}}\left[\Delta \hat{\rho}-\Delta \hat{k}\left(1-\frac{s_{\text {eff }}^{2}}{c_{\text {eff }}^{2}}\right)\right] \\
& -\frac{e^{2}}{s_{\text {eff }}^{2}} x_{t}\left[2 \Delta \hat{\rho}-(\Delta \hat{\rho})_{l e a d}-\hat{f}+\Delta \hat{k} \frac{s_{\text {eff }}^{2}}{c_{\text {eff }}^{2}}\right],
\end{aligned}
$$


where

$\Delta \hat{\rho} \equiv \operatorname{Re}\left[A_{W W}\left(M_{W}^{2}\right)-\hat{c}^{2} A_{Z Z}\left(M_{Z}^{2}\right)\right] / M_{W}^{2}$, $x_{t}=3 G_{F} M_{t}^{2} /\left(8 \sqrt{2} \pi^{2}\right), \Delta \hat{r}_{W}=-2 \delta e / e+$ $\left(e^{2} / \hat{s}^{2}\right) \hat{f}, \quad(\Delta \hat{\rho})_{l e a d}=\left(3 / 64 \pi^{2}\right) M_{t}^{2} / M_{W}^{2}$, $\hat{f} \equiv\left(\operatorname{Re} A_{W W}\left(M_{W}^{2}\right)-A_{W W}(0)\right) / M_{W}^{2}+V_{W}+$ $M_{W}^{2} B_{W}, A_{W W}$ and $A_{Z Z}$ are the $W$ and $Z$ selfenergies modulo a factor $\hat{e}^{2} / \hat{s}^{2}, V_{W}$ and $B_{W}$ are vertex and box corrections contributing to $\mu$ decay, and $\delta e$ stands for the charge renormalization counterterm. It is understood that, in Eq. (3), $\hat{s}^{2}$ is replaced everywhere by $s_{e f f}^{2}$. The corrections $\Delta \hat{r}_{W}, \Delta \hat{\rho}, \Delta \hat{k}$, and $\hat{f}$ depend also on $c^{2}=M_{W}^{2} / M_{Z}^{2}$. In order to obtain an expression that depends solely on $c_{e f f}^{2}=1-s_{e f f}^{2}, M_{W}^{2}$ is replaced by $c^{2} M_{Z}^{2}$; in two-loop contributions, $c^{2}$ is replaced by $c_{e f f}^{2}$, since the difference is of third order; in one-loop corrections, a Taylor expansion about $c^{2}=c_{e f f}^{2}$ is made, in conjunction with the one-loop expression for $c^{2}-c_{e f f}^{2}$. The corresponding expression for $M_{W}$ is given in Ref. [6]. An interesting feature is that the calculation of $s_{e f f}^{2}$ is completely decoupled from that of $M_{W}$, while the $s_{\text {eff }}^{2}$ results are employed to calculate $M_{W}$. The results for the leptonic partial widths $\Gamma_{l}$ of the $Z$ have been recently obtained [12]. A detailed comparison shows that, for $M_{H}=100 \mathrm{GeV}$, the difference $\left|s_{e f f}^{2}(\overline{M S})-s_{e f f}^{2}(E F F)\right|$ of the $\overline{M S}$ and EFF calculation of $s_{e f f}^{2}$ is $\leq 10^{-5}$ over the range $30 \mathrm{GeV} \leq \mu \leq 200 \mathrm{GeV}$ and exhibits a maximum at $\mu \approx 70 \mathrm{GeV}$. In the $M_{W}$ case, one finds $\left|M_{W}(\overline{M S})-M_{W}(E F F)\right| \leq 1 \mathrm{MeV}$ over the range $50 \mathrm{GeV} \leq \mu \leq 205 \mathrm{GeV}$ and a maximum at $\mu \approx 100 \mathrm{GeV}$. At $\mu=300 \mathrm{GeV}$, the differences amount to $\approx 3 \times 10^{-5}$ and $3 \mathrm{MeV}$, respectively. These findings give support to the usual choice $\mu=M_{Z}$ in the $\overline{M S}$ calculations of observables in the resonance region. It should be pointed out, however, that this satisfactory state of affairs holds when the corrections of $\mathcal{O}\left(\alpha^{2}\left(M_{t} / M_{W}\right)^{2}\right)$ are included. If these contributions are excluded, $M_{W}(\overline{M S})$ is a monotonically decreasing function of $\mu$ over the range $30 \mathrm{GeV} \leq \mu \leq 500 \mathrm{GeV}$ [13], and the choice of scale is very ambiguous. In summary, the EFF approach has the virtue of eliminating the scale ambiguity which, in some cases, may create a significant theoretical uncertainty.

\section{Evidence for Electroweak Bosonic Cor- rections}

It turns out that $\Delta r_{\text {eff }}$ is very sensitive to electroweak bosonic contributions (EWBC), i.e. corrections involving virtual bosons: $W$ 's, $Z$, $H$, $\phi$ 's. They are subleading numerically, but very important conceptually! One way to obtain sharp evidence for these corrections is to measure $\Delta r_{\text {eff }}$. Using the current experimental value $\left(s_{e f f}^{2}\right)_{\exp }=0.23149 \pm 0.00017$ and Eq.(1), we find $\left(\Delta r_{e f f}\right)_{\exp }=0.06047 \pm 0.00048$. On the other hand, subtracting the EWBC, the theoretical evaluation leads to: $\left(\Delta r_{\text {eff }}\right)_{\text {theor }}^{\text {subtr }}=0.05106 \pm$ 0.00083 . The difference is $0.00941 \pm 0.00096$, thus providing evidence for the presence of EWBC at the $9.8 \sigma$ level $[5,8,14]$ !

\section{The Higgs-less Scenario}

The corrections $\Delta r_{\text {eff }}$ and $\Delta r$ have been also employed to discuss the scale of new physics in a hypothetical scenario in which the Higgs boson is absent [15]. At the one-loop level, the Higgs boson contribution to $\Delta r_{e f f}$ is a complicated function of $\xi=M_{H}^{2} / M_{Z}^{2}$, given in Ref. [15]. It may be written in the form

$$
\begin{aligned}
& \left(\Delta r_{e f f}\right)_{H}=\frac{\alpha}{4 \pi \hat{s}^{2} \hat{c}^{2}}\left(\frac{5}{3}-\frac{3}{2} c^{2}\right) \\
& \times\left(\frac{1}{n-4}+C+\ln \frac{M_{Z}}{\mu}\right)+\left(\Delta r_{e f f}\right)_{H}^{\overline{M S}},
\end{aligned}
$$

where the first term is the divergent part and the second one is the $\overline{M S}$-renormalized contribution evaluated at $\mu=M_{Z}(C=[\gamma-\ln 4 \pi] / 2$, $\mu=$ 't Hooft scale). Subtracting $\left(\Delta r_{e f f}\right)_{H}$ from $\Delta r_{\text {eff }}$ we have

$$
\begin{aligned}
& \Delta r_{e f f}-\left(\Delta r_{e f f}\right)_{H}=\Delta r_{e f f}-\left(\Delta r_{e f f}\right)_{H}^{\overline{M S}} \\
& -\frac{\alpha}{4 \pi \hat{s}^{2} \hat{c}^{2}}\left(\frac{5}{3}-\frac{3}{2} c^{2}\right)\left(\frac{1}{n-4}+C+\ln \frac{M_{Z}}{\mu}\right) .
\end{aligned}
$$

Clearly, Eq.(5) is divergent and scale dependent. We now conjecture that contributions from unknown new physics (NP) cancel the divergence and scale dependence of Eq.(5). Thus, the NP contribution to $\Delta r_{\text {eff }}$ must be of the form:

$$
X=\frac{\alpha}{4 \pi \hat{s}^{2} \hat{c}^{2}}\left(\frac{5}{3}-\frac{3}{2} c^{2}\right)\left(\frac{1}{n-4}+C+\ln \frac{M}{\mu}\right) \text {. }
$$


We note that in the $\overline{M S}$ renormalization approach, the term proportional to $\ln M / \mu$ represents the NP contribution to $\Delta r_{\text {eff }}$ at scale $\mu$. If the NP is characterized by a scale $\Lambda$, we may decompose

$\ln \frac{M}{\mu}=\ln \frac{\Lambda}{\mu}+K$,

where the term involving $K \equiv \ln \frac{M}{\Lambda}$ represents the NP contribution to $\Delta r_{\text {eff }}$ at scale $\Lambda$. Adding $X$ to $\Delta r_{e f f}-\left(\Delta r_{e f f}\right)_{H}$ we find the expression for $\Delta r_{e f f}$ in the new scenario (NS) in which the Higgs boson contribution has been replaced by new physics:

$$
\begin{aligned}
\left(\Delta r_{e f f}\right)_{N S} & =\Delta r_{e f f}-\left(\Delta r_{e f f}\right)_{H}^{\overline{M S}} \\
& +\frac{\alpha}{4 \pi \hat{s}^{2} \hat{c}^{2}}\left(\frac{5}{3}-\frac{3}{2} c^{2}\right) \ln \frac{M}{M_{Z}}
\end{aligned}
$$

The last term represents the NP contribution to $\Delta r_{e f f}$ at scale $M_{Z}$. Calculating $\Delta r_{e f f}-\left(\Delta r_{e f f}\right)_{H}^{\overline{M S}}$ and equating $\left(\Delta r_{e f f}\right)_{N S}=$ $\left(\Delta r_{\text {eff }}\right)_{\text {exp }}$, we can determine $\ln \frac{M}{M_{Z}}$. Employing $\Delta \alpha_{h}^{(5)}=0.02761 \pm 0.00036$ and the other experimental inputs, one finds

$\ln \frac{M}{M_{Z}}=0.307 \pm 0.485$,

which corresponds to a central value $M_{c}=$ $124 \mathrm{GeV}$ and a $95 \%$ CL upper bound $M^{95}=$ $275 \mathrm{GeV}$. If the model-dependent constant $K$ is positive, we see from Eq.(7) that $\Lambda$ is sharply bounded: $\Lambda \leq 275 \mathrm{GeV} @ 95 \% \mathrm{CL}$. Instead, if $K<0, \Lambda$ is not bounded by these considerations. Thus, we can group the NP models into two classes, according to the sign of $K$. Furthermore, if for instance $\Lambda=1 \mathrm{TeV}$, we have $\ln \frac{\Lambda}{M_{Z}}=2.395$ and we find from Eqs. $(7,9)$ that $K=-2.088 \pm 0.435$. Thus, for such $\Lambda$ values, a substantial cancellation of logarithmic and constant terms is required $[15,16]$. Similar results are obtained from the corresponding analysis of $\Delta r[15]$.

\section{Simple formulae for $s_{e f f}^{2}, M_{W}, \Gamma_{l}$}

Simple formulae that reproduce accurately the numerical results of the codes in the range
$20 \mathrm{GeV} \leq M_{H} \leq 300 \mathrm{GeV}$, probed by recent experiments, have been presented [12]. They are of the form:

$$
\begin{aligned}
s_{\text {eff }}^{2}= & \left(s_{\text {eff }}^{2}\right)_{0}+c_{1} A_{1}+c_{5} A_{1}^{2}+c_{2} A_{2} \\
& -c_{3} A_{3}+c_{4} A_{4}, \\
M_{W}= & M_{W}^{0}-d_{1} A_{1}-d_{5} A_{1}^{2}-d_{2} A_{2} \\
& +d_{3} A_{3}-d_{4} A_{4} \\
\Gamma_{l}= & \Gamma_{l}^{0}-g_{1} A_{1}-g_{5} A_{1}^{2}-g_{2} A_{2} \\
& +g_{3} A_{3}-g_{4} A_{4},
\end{aligned}
$$

where $A_{1} \equiv \ln \left(M_{H} / 100 \mathrm{GeV}\right), \quad A_{2} \equiv$ $\left[\Delta \alpha_{h}^{(5)} / 0.02761\right]-1, A_{3} \equiv\left(M_{t} / 174.3 \mathrm{GeV}\right)^{2}-1$, $A_{4} \equiv\left[\alpha_{s}\left(M_{Z}\right) / 0.118\right]-1$. The constants $c_{i}, d_{i}$, $g_{i}(i=1-5)$ are given in Ref. [12] for the EFF, $\overline{M S}$, and OS schemes of renormalization. Furthermore, Eqs.(10-12) retain their accuracy over the range of $\Delta \alpha_{h}^{(5)}$ results from recent calculations. Using Eq. (10) in the EFF scheme and $\left(s_{\text {eff }}^{2}\right)_{\text {exp }}=0.23149 \pm 0.00017$, one finds $M_{H}=$ $124_{-52}^{+82} \mathrm{GeV}$ and a $95 \%$ CL upper bound $M_{H}^{95}=$ $280 \mathrm{GeV}$. Instead, Eq. (11) and $\left(M_{W}\right)_{\text {exp }}=$ $80.451 \pm 0.033 \mathrm{GeV}$ lead to $M_{H}=23_{-23}^{+49} \mathrm{GeV}$, $M_{H}^{95}=122 \mathrm{GeV}$. Thus, $M_{W}$ constrains $M_{H}$ much more sharply than $s_{e f f}^{2}$ ! It is important to note that the $M_{H}^{95}$ value derived from $M_{W}$, and the direct exclusion bound $M_{H}>114 \mathrm{GeV} @ 95 \%$ $\mathrm{CL}$, suggest a very narrow window for $M_{H}$ ! One may also extract $A_{1}$ from $\left(s_{\text {eff }}^{2}\right)_{\text {exp }}$ and Eq. (10), to predict $M_{W}$ via Eq. (11): $\left(M_{W}\right)_{\text {indir. }}=$ $80.374 \pm 0.025 \mathrm{GeV}$, which is close to the corresponding value $\left(M_{W}\right)_{\text {indir. }}=80.379 \pm 0.023 \mathrm{GeV}$ obtained in the global analysis [17], and differs from $\left(M_{W}\right)_{\text {exp }}$. by $1.86 \sigma$. Finally, we may use simultaneously Eq. (10-12) in conjunction with $\left(s_{\text {eff }}^{2}\right)_{e x p},\left(M_{W}\right)_{e x p}$, and $\left(\Gamma_{l}\right)_{e x p}$ to obtain $M_{H}=$ $97_{-41}^{+66} \mathrm{GeV}, M_{H}^{95}=223 \mathrm{GeV}$, to be compared with $M_{H}=85_{-34}^{+54} \mathrm{GeV}, M_{H}^{95}=196 \mathrm{GeV}$ in the recent EWWG fit.

The current determination of $\left(s_{\text {eff }}^{2}\right)_{\text {exp }}$ has $\chi^{2} /$ d.o.f. $=10.6 / 5$, corresponding to a CL of only $6 \%$, and shows an intriguing dichotomy: the leptonic observables $\left(A_{l}(S L D), A_{l}\left(P_{\tau}\right), A_{f b}^{(0, l)}\right)$ lead to $\left(s_{\text {eff }}^{2}\right)_{l}=0.23113 \pm 0.00021$, while the value from the hadronic ones $\left(A_{f b}^{(0, b)}, A_{f b}^{(0, c)},<Q_{f b}>\right)$ is $\left(s_{\text {eff }}^{2}\right)_{h}=0.23220 \pm 0.00029$. Thus, there 
is a $3 \sigma$ difference between the two determinations! Furthermore, from $\left(s_{\text {eff }}^{2}\right)_{l}$ one finds $M_{H}=$ $59_{-29}^{+50} \mathrm{GeV}, M_{H}^{95}=158 \mathrm{GeV}$, closer to the result from $\left(M_{W}\right)_{\text {exp }}$. If $\left(s_{\text {eff }}^{2}\right)_{l}-\left(s_{\text {eff }}^{2}\right)_{h}$ reflects a statistical fluctuation, one possibility is to enlarge the error by $\left[\chi^{2} / \text { d.o.f. }\right]^{1 / 2}$ (PDG prescription), leading to $s_{\text {eff }}^{2}=0.23149 \pm 0.00025$. Interestingly, increasing the error in $s_{\text {eff }}^{2}$ leads to smaller $M_{H}^{95}$ in the combined $s_{e f f^{-}}^{2} M_{W}-\Gamma_{l}$ analysis: $223 \mathrm{GeV} \rightarrow 201 \mathrm{GeV}$ ! The reason is that this procedure gives enhanced weight to the $M_{W}$ input, which prefers a smaller $M_{H}$. If $\left(s_{e f f}^{2}\right)_{l}-\left(s_{\text {eff }}^{2}\right)_{h}$ is due to new physics involving the $(t, b)$ generation, a substantial, tree-level change in the $Z b_{R} \bar{b}_{R}$ coupling is required [18]. If the discrepancy were to settle on the leptonic side, a scenario with light $\tilde{\nu}$ and $\tilde{g}$ would improve the agreement with the electroweak data and the direct lower bound on $M_{H}$ [19].

It has been pointed out by several people that, if the central values of $M_{t}$ and $M_{W}$ remain as they are now, but the errors shrink sharply as expected at Tevatron/LHC or even much better at LC + GigaZ, a discrepancy would be established with the SM, that can be accommodated in the MSSM!

The comparison of the calculations of $s_{e f f}^{2}, M_{W}$, and $\Gamma_{l}$ in the EFF, $\overline{M S}$, and OS frameworks has been applied to study the scheme dependence and to estimate the theoretical error arising from the truncation of the perturbative series [12]. Including QCD uncertainties, the theoretical errors have been estimated to be $\delta s_{\text {eff }}^{2} \approx 6 \times 10^{-5}$ and $\delta M_{W} \approx 7 \mathrm{MeV}$.

\section{Field Renormalization Constant for Un- stable Particles}

In Ref. [20] it was found that, in the gauge theory context, the conventional definitions of mass and width of unstable particles are gauge dependent in next-to-next-to-leading order (NNLO). Furthermore, the conventional expression for the field renormalization constant tends to 0 as the particle mass approaches from below some physical thresholds, which implies the absurd conclusion that, in such a case, all decays are forbidden.
In Ref. [20], it was proposed that the first problem can be solved by considering the complex valued position $\bar{s}$ of the propagator's pole, which is gauge invariant. We have: $\bar{s}=M_{o}^{2}+A(\bar{s})$, where $M_{o}$ is the bare mass and $A(s)$ the self-energy. Decomposing $\bar{s}=m_{2}^{2}-i m_{2} \Gamma_{2}$, where $m_{2}$ and $\Gamma_{2}$ are real, one identifies $m_{2}$ and $\Gamma_{2}$ with the mass and width of the particle:

$m_{2}^{2}=M_{o}^{2}+\operatorname{Re} A(\bar{s})$,

$m_{2} \Gamma_{2}=-\operatorname{Im} A(\bar{s})$.

In Ref.[21], it was proposed that the second problem can be solved by defining the field renormalization constant $\hat{Z}$ by means of the normalization condition

$m_{2} \Gamma_{2}=-\hat{Z} \operatorname{Im} A\left(m_{2}^{2}\right)$,

which, in conjunction with Eq. (14), leads to

$\hat{Z}^{-1}=1+\frac{\operatorname{Im}\left(A(\bar{s})-A\left(m_{2}^{2}\right)\right)}{m_{2} \Gamma_{2}}$.

In the narrow width approximation, the r.h.s. of Eq. (16) becomes $1-\operatorname{Re} A^{\prime}\left(m_{2}^{2}\right) \approx 1-\operatorname{Re} A^{\prime}\left(M^{2}\right)$, where $M^{2}$ is the on-shell mass, and $\hat{Z}$ reduces to the conventional expression. We note that: i) $\hat{Z}$, defined by Eq. (16), involves a finite difference, rather than a derivative, thus avoiding the threshold problem; ii) using Eq. (16) we see that the r.h.s. of Eq. (15) is gauge invariant, since it equals $m_{2} \Gamma_{2}$ as a mathematical identity. It was also shown that the use of Eq. (16) removes unphysical threshold singularities in the relation between on-shell and pole widths [22]. This approach has been recently discussed in the framework of renormalization theory $[23,24]$. Dividing the unrenormalized transverse propagator $-i Q_{\mu \nu}\left[s-M_{o}^{2}-A(s)\right]^{-1}\left(Q_{\mu \nu}=g_{\mu \nu}-q_{\mu} q_{\nu} / q^{2}\right)$ by $\hat{Z}$, and introducing $S(s) \equiv \hat{Z} A(s), \delta M^{2} \equiv$ $\operatorname{Re} S(\bar{s}), \hat{Z} \equiv 1-\delta \hat{Z}$, we obtain the renormalized propagator:

$\mathcal{D}=-i Q_{\mu \nu} /\left(s-m_{2}^{2}-S^{(r)}(s)\right)$,

where

$S^{(r)}(s)=S(s)-\delta M^{2}+\delta \hat{Z}\left(s-m_{2}^{2}\right)$

stands for the renormalized self-energy. Since $\delta M^{2}$ and $\delta \hat{Z}$ are real, they should be chosen so 
that $\operatorname{Re} S^{(r)}(s)$ is ultraviolet convergent to all orders. Once this is done, $\operatorname{Im} S^{(r)}(s)=\operatorname{Im} S(s)=$ $\hat{Z}$ Im $A(s)$ must also be convergent, since there are no additional counterterms available. This means that $\hat{Z}$ may be defined by imposing an appropriate normalization condition on $\operatorname{Im} S(s)$. A particularly simple one is

$\operatorname{Im} S\left(m_{2}^{2}\right)=-m_{2} \Gamma_{2}$,

which coincides with Eq. (15)! It was proposed independently in Ref. [21] to solve the threshold and gauge-dependence problems, and in Ref. [24] to implement a systematic order by order removal of the ultraviolet divergences in $S^{(r)}(s)$. In Ref. [23] it was also emphasized that in this formulation one can derive closed and exact expressions for the mass and field-renormalization counterterms, to wit

$\delta M^{2}=\operatorname{Re} S(\bar{s}) ; \delta \hat{Z}=\frac{\operatorname{Im}\left[S(\bar{s})-S\left(m_{2}^{2}\right)\right]}{m_{2} \Gamma_{2}}$.

In many cases, $\Gamma_{2}=\mathcal{O}\left(g^{2}\right)$, where $g$ is a generic gauge coupling. If $\delta M^{2}$ and $\delta \hat{Z}$ admit expansions in powers of $\Gamma_{2}$, they can be expressed as series involving $R \equiv \operatorname{Re} S(s), I \equiv \operatorname{Im} S(s)$, and their powers and derivatives evaluated at $s=m_{2}^{2}$. These expansions of Eq. (20) coincide with the order by order analysis in Ref. [24]. However, in other important instances, such as the photonic corrections to the $W$ self-energy, such expansions are ill-defined and lead to power-like infrared divergences! In such cases one should employ the exact formulae in Eq. (20), which lead to sensible expressions for $\delta M^{2}, \delta \hat{Z}$, and the renormalized propagator [23].

\section{REFERENCES}

1. A. Sirlin, Phys. Rev. D22, 971 (1980).

2. K. I. Aoki, Z. Hioki, M. Konuma, R. Kawabe and T. Muta, Prog. Theor. Phys. Suppl. 73, 1 (1982).

3. A. Hollik, Fortsh. Phys. 38, 165 (1990), and refs. cited therein.

4. See, for example, G. Degrassi, S. Fanchiotti and A. Sirlin, Nucl. Phys. B351, 49 (1991), and refs. cited therein.
5. A. Sirlin, hep-ph/0209079, to be published in Journal of Physics G.

6. A. Ferroglia, G. Ossola and A. Sirlin, Phys. Lett. B507, 147 (2001).

7. A. Ferroglia, G. Ossola and A. Sirlin, Novel Approach to Renormalize the Electroweak Sector of the Standard Model, Contribution to the 20th International Symposium on Lepton and Photon Interactions at High Energies, Rome, Italy, July 2001, arXiv:hepph/0106094.

8. A. Sirlin, Proc. of the 19th Intl. Symp. on Photon and Lepton Interactions at High Energy LP99, ed. J.A. Jaros and M.E. Peskin, 398 (2000).

9. G. Degrassi, P. Gambino and A. Vicini, Phys. Lett. B 383, 219 (1996).

10. G. Degrassi, P. Gambino and A. Sirlin, Phys. Lett. B394, 188 (1997).

11. P. Gambino and A. Sirlin, Phys. Rev. D49, R1160 (1994).

12. A. Ferroglia, G. Ossola, M. Passera and A. Sirlin, Phys. Rev. D65, 113002 (2002).

13. G. Degrassi, P. Gambino, M. Passera, and A. Sirlin, Phys. Lett. B418, 209 (1998).

14. P. Gambino and A. Sirlin, Phys. Rev. Lett. 73, 621 (1994).

15. B. A. Kniehl and A. Sirlin, Eur. Phys. J. C16 635 (2000).

16. G. Altarelli, arXiv:hep-ph/9912291.

17. G. Myatt, LEP and SLD Electroweak Working Group, Preliminary [03-15-02].

18. W.J.Marciano, Phys. Rev. D 60, 093006 (1999); M. S. Chanowitz, Report LBNL43248, arXiv:hep-ph/9905478.

19. G. Altarelli, F. Caravaglios, G. F. Giudice, P. Gambino and G. Ridolfi, JHEP 0106, 018 (2001).

20. A. Sirlin, Phys. Rev. Lett. 67, 2127 (1991).

21. B. A. Kniehl, C. P. Palisoc and A. Sirlin, Nucl. Phys. B 591, 296 (2000).

22. B. A. Kniehl, C. P. Palisoc and A. Sirlin, Phys. Rev. D 66, 057902 (2002).

23. B. A. Kniehl and A. Sirlin, Phys. Lett. B 530, 129 (2002).

24. M. L. Nekrasov, Phys. Lett. B 531, 225 (2002). 